Check for updates

Cite this: RSC Adv., 2017, 7, 28494

\title{
Investigation of the hole transport characterization and mechanisms in co-evaporated organic semiconductor mixtures
}

\author{
Yanping Wang, (D) *a Qingcheng Liang, ${ }^{a}$ Jinying Huang, ${ }^{\mathrm{b}}$ Dongge Ma (D) ${ }^{\mathrm{bc}}$ \\ and Yurong $\mathrm{Jiao}^{\mathrm{d}}$
}

The hole transport characteristics in small molecule semiconductor mixtures of HAT-CN : NPB, HAT$\mathrm{CN}$ : TAPC and HAT-CN : CBP in the ratio of $2: 1$ have been investigated by admittance spectroscopy measurements. It is found that the hole mobility $\left(10^{-5}-10^{-4} \mathrm{~cm}^{2} \mathrm{~V}^{-1} \mathrm{~s}^{-1}\right)$ variation with electric field in HAT-CN : NPB is the same as that in HAT-CN : TAPC. The hole transport in HAT-CN : CBP is spacecharge-limited current (SCLC) with exponential distribution traps, different from SCLC with free trap distributions of HAT-CN : NPB and HAT-CN : TAPC mixtures. The hole mobility in the HAT-CN : CBP mixture is obtained as $10^{-7}-10^{-5} \mathrm{~cm}^{2} \mathrm{~V}^{-1} \mathrm{~s}^{-1}$, which is one or two orders of magnitude lower than those of the other two mixtures. It can be seen that the mobility exhibits strong field-dependence in low electric field region. The mobility slightly increases as the electric field increases and almost saturates in high electric field region. It is shown that the trapping effect leads to the much lower hole mobility. The hole transport mechanisms in the three mixtures have been further studied through energy level analysis and atomic force microscopy.

Received 7th May 2017

Accepted 25th May 2017

DOI: $10.1039 / \mathrm{c} 7 \mathrm{ra05131j}$

rsc.li/rsc-advances
So far the charge carrier transport characteristics in organic semiconductors have been usually analyzed by several techniques, such as time of flight (TOF), ${ }^{\mathbf{9}, \mathbf{1 0}}$ transient electroluminescence (TEL), ${ }^{\mathbf{1 1}, 12}$ space charge limited current (SCLC) ${ }^{13-15}$ and admittance spectroscopy (AS). ${ }^{16-21}$ In these techniques, notably, AS has been shown to be a feasible and powerful tool to explore the charge carrier transport kinetics and relaxation processes and involved in optoelectronic devices. In addition, it is applicable to charge carrier transport characteristics of organic semiconductor film whose thickness is approximately comparable to that of practical devices. It can also be applied without the need for a complex experimental setup.

The 1,4,5,8,9 and 11-hexaazatriphenylene-hexacarbonitrile (HAT-CN) material has been drawing a great deal of attention because it has a lot of interesting characteristics. ${ }^{22-28}$ It has been adapted as the charge generation layer in some tandem OLEDs for higher power efficiency due to its very good electron affinity. It has also been used to modify metal surface electronic properties due to its unoccupied molecular orbital (LUMO) almost close to the Fermi level. However, there are rare reports about the study on HAT-CN organic mixtures by AS measurements. In this work, we systematically investigated the hole transport characteristics and mechanisms in the mixtures of HATCN : NPB, HAT-CN : TAPC and HAT-CN : CBP by current density-voltage characteristics, AS technique, energy level analysis and atomic force microscopy. 


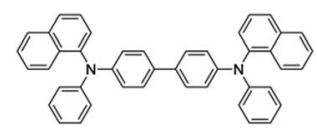

NPB

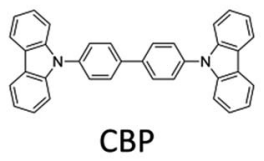

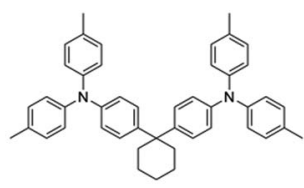

TAPC

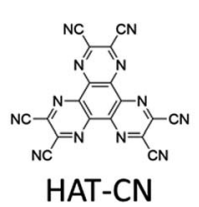

HAT-CN
Fig. 1 Structures of organic molecules used in this work.

\section{Experimental}

The hole-only devices were fabricated on glass substrates precoated with a $180 \mathrm{~nm}$-thick indium tin oxide (ITO) film with a sheet resistance of $\sim 10 \Omega$ per square. Before the fabrication process, the ITO surface was thoroughly cleaned in an ultrasonic bath using subsequently detergents and deionized water, dried at $120{ }^{\circ} \mathrm{C}$. The device structure was ITO/HAT-CN $(10 \mathrm{~nm}) / \mathrm{HAT}-$ $\mathrm{CN}: \mathrm{X}(2: 1,180 \mathrm{~nm}) / \mathrm{HAT}-\mathrm{CN}(10 \mathrm{~nm}) / \mathrm{Al}$. Here, $\mathrm{X}$ was $N, N^{\prime}$ di(naphthalene-1-yl)-N, $N^{\prime}$-diphenyl-benzidine (NPB), 4,4'-cyclohexylidenebis[ $N, N$-bis(4-methylphenyl)benze namine] (TAPC) and 4,4'-bis(carbazol-9-yl)-biphenyl (CBP), respectively. Structures of organic molecules are depicted in Fig. 1. All organic materials were grown in succession by thermal evaporation in a high-vacuum $\left(\sim 4 \times 10^{-4} \mathrm{~Pa}\right)$ without breaking vacuum. The $\mathrm{Al}$ electrodes were deposited on the substrates through shadow masks. The active area of device is $4 \times 4 \mathrm{~mm}^{2}$. During the deposition process, the rate was controlled with quartz crystal oscillators and monitored by a frequency counter and calibrated by a Dektak $6 \mathrm{M}$ profiler (Veeco).

The current density-voltage $(J-V)$ characteristics were carried out using a Keithley 2400 sourcemeter integrated with a vacuum cryostat (Optistat DN-V, Oxford Instruments). The admittance was measured at room temperature by an Agilent E4980A precision LCR meter in the frequency range of $20 \mathrm{~Hz}-13 \mathrm{MHz}$ with the oscillation amplitude of the ac voltage kept at $100 \mathrm{mV}$. The morphologies of the thin films on ITO substrates were tested by atomic force microscopy (AFM) (SPA 400, Seiko Instruments Inc.).

\section{Results and discussion}

\subsection{Current density-voltage characteristics}

The $J-V$ characteristics of the hole-only devices based on HATCN : NPB, HAT-CN : TAPC and HAT-CN : CBP mixtures are shown in Fig. 2. At a constant current density, the driving voltages of HAT-CN : NPB and HAT-CN : TAPC mixtures are close and lower, demonstrating the similar hole transport ability. In our previous work, ${ }^{29}$ the $J-V$ characteristics of the two devices with different mixing concentration $(y)$ have been discussed in detail. The results showed that their hole conductions $(y<90 \%)$ exhibit space-charge-limited current

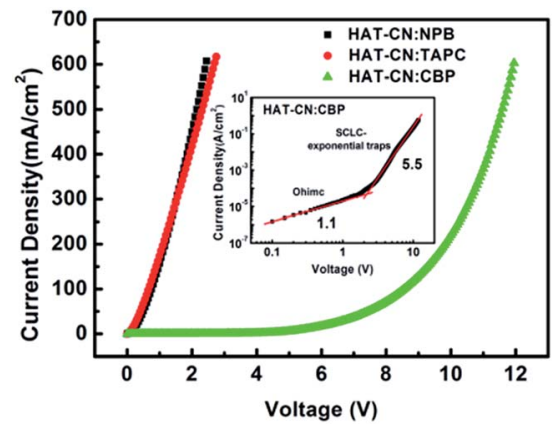

Fig. 2 The current-voltage characteristics of the hole-only devices based on HAT-CN : NPB, HAT-CN : TAPC and HAT-CN : CBP mixtures. Inset: the current-voltage characteristics for HAT-CN : CBP device in double logarithmic representation.

(SCLC) with free trap distributions. Conversely, it is shown a higher voltage for HAT-CN : CBP mixture. As shown in the inset, it clearly show two distinct regions: (1) ohmic region, the slope of $\log J-\log V$ curve is about 1.1 at the lower voltage region. (2) SCLC with exponential trap distribution region, the slope of $J-V$ curve in double logarithmic is about 5.5 at higher voltages. For organic semiconductor devices, the charge carrier mobility plays a crucial role in determining the $J-V$ characteristics.

In order to clearly demonstrate the similarities and differences in the three hole-only devices, the hole mobilities have been determined by using frequency-dependent AS.

\subsection{Mobility characteristics}

AS is technique based on the measurement of the frequencydependent capacitance of an organic thin film. For a twoterminal device consisting of an organic semiconductor with a dielectric constant $\varepsilon$ and a thickness $d$, holes are injected from the anode when it is applied a forward dc bias voltage $\left(V_{\mathrm{dc}}\right)$. In the case of application of a small ac signal (modulation), with an angular frequency $\omega=2 \pi f$, the time-dependent injected charge current density $j(t)$ can be given by: ${ }^{30}$

$$
\begin{aligned}
j(t)= & q \mu(t) \rho_{\mathrm{dc}}(x) E_{\mathrm{ac}}(x, t)+\varepsilon \varepsilon_{0} \mu(t) E_{\mathrm{dc}}(x) \frac{\partial E_{\mathrm{ac}}(x, t)}{\partial x} \\
& +\varepsilon \varepsilon_{0} \frac{\partial E_{\mathrm{ac}}(x, t)}{\partial t}
\end{aligned}
$$

where $q$ is the electron charge, $\rho_{\mathrm{dc}}(x)$ is the dc charge density, $\mu(t)$ is the time dependent mobility, $E_{\mathrm{dc}}(x)$ and $E_{\mathrm{ac}}(x)$ is the dc and ac electric field components, respectively. According to eqn (1), there are three different contributions to $j(t)$ : (1) the response of $\rho_{\mathrm{dc}}(x)$ in the device. (2) The current due to the additional time-dependent injected charge density. (3) The displacement current by charge relaxation. The complex admittance $Y(\Omega)$ is related to $i_{\mathrm{ac}}$ and $v_{\mathrm{ac}}$ as follows: $Y(\Omega)=\frac{i_{\mathrm{ac}}}{v_{\mathrm{ac}}}$, where $G, C$ and $i$ are the conductance, capacitance and imaginary unit, respectively. It is shown that $Y(\Omega)$ can be obtained by applying a Fourier transform to eqn (1): 


$$
\begin{aligned}
& Y(\Omega)=G(\omega)+i B(\omega)=G(\omega)+i \omega C(\omega)= \\
& \frac{\varepsilon \varepsilon_{0} A}{\tau_{\mathrm{dc}} d}\left\{\frac{\Omega^{3}}{2 i[0.75 \tilde{\mu}(\Omega)]^{2}\left[1-\exp \left(\frac{-i 4(\Omega)}{3 \tilde{\mu}(\Omega)}\right)\right]+1.5 \tilde{\mu}(\Omega) \Omega-i \Omega^{2}}\right\}
\end{aligned}
$$

where $A$ and $\varepsilon_{0}$ are the area of the sample and the permittivity of the free space, respectively. In the above equation, $\tau_{\mathrm{dc}}$ is the charge carrier average transit time across the organic thin film in the absence of the ac signal, which is directly related to the energy disorder of organic thin film, and the normalized frequency is defined as $\Omega=\omega \tau_{\mathrm{dc}}$. The frequency-dependent capacitance can be re-plotted as the negative differential susceptance $-\Delta B=-2 \pi f\left(C-C_{\text {geo }}\right)$, where the geometrical capacitance $C_{\text {geo }}=\varepsilon \varepsilon_{0} A / d$. The curve typically yields a maximum of $-\Delta B$ at a characteristic frequency $f_{\mathrm{r}}=0.56 / \tau_{\mathrm{dc}}$. The carrier mobility can be then extracted by,

$$
\mu_{\mathrm{dc}}=\frac{f_{\mathrm{r}} d^{2}}{0.56\left(V-V_{\mathrm{bi}}\right)}
$$

where $V_{\mathrm{bi}}$ is the built-in potential.

Fig. 3(a) shows $C-f$ curves for the hole-only devices base on HAT-CN : NPB, HAT-CN : TAPC and HAT-CN : CBP mixtures at different applied bias voltages. In the low or intermediate frequency regions, clearly, the capacitances of the three devices decreased with frequency increasing. The reasons are as follows. When the ac-modulation is lower than the characteristic trapping-detrapping time constant, the trap states begin to respond and induce additional capacitive contribution. At further higher frequency, conversely, the trap states cannot follow the ac modulation, the capacitance drops to a plateau, leaving the geometrical capacitance $\left(C_{\text {geo }}\right)$. Toward high frequency, the capacitance becomes little lower than the $C_{\text {geo }}$, which is determined by the transit time. ${ }^{31}$ In the frequency range higher than the $f_{\mathrm{r}}$, the capacitance comes close to the $C_{\text {geo }}$
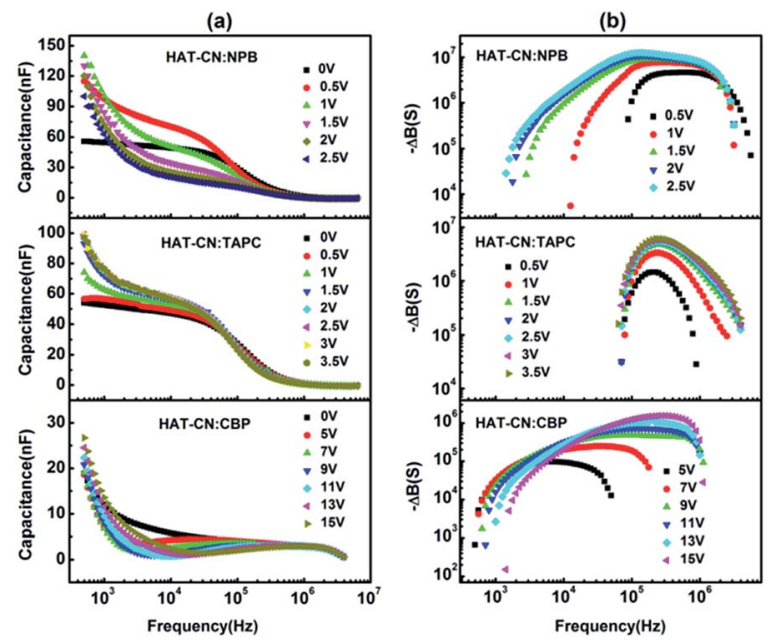

Fig. 3 (a) Frequency dependence of capacitance of HAT-CN : NPB, HAT-CN : TAPC and HAT-CN : CBP devices at different applied bias voltages. (b) Negative differential susceptance $-\Delta B$ dependent frequency of three devices with different applied bias voltages. because the period of the ac electric field is too short to redistribute the space charges in the mixture devices. As shown in Fig. 3(b), a clear maximum in $-\Delta B-f$ occurs at a well defined frequency $f_{\mathrm{r}}$ for each device. On the base of eqn (3), the hole mobilities can be calculated, which are summarized in Fig. 4.

For HAT-CN : NPB and HAT-CN : TAPC mixtures, the mobilities are in the range of $4.1 \times 10^{-5}-6.5 \times 10^{-4} \mathrm{~cm}^{2} \mathrm{~V}^{-1} \mathrm{~s}^{-1}$ and $5.8 \times 10^{-5}-2.3 \times 10^{-4} \mathrm{~cm}^{2} \mathrm{~V}^{-1} \mathrm{~s}^{-1}$, respectively. As expected from the $J-V$ characteristics of the hole-only devices, HAT-CN : NPB and HAT-CN : TAPC have similar and higher hole mobilities. At the same electric field, the mobility $\left(\sim 10^{-4}\right.$ $\mathrm{cm}^{2} \mathrm{~V}^{-1} \mathrm{~s}^{-1}$ ) of the two mixtures calculated by AS measurements is one order of magnitude smaller than our previous result $\left(\sim 10^{-3} \mathrm{~cm}^{2} \mathrm{~V}^{-1} \mathrm{~s}^{-1}\right)^{29}$ obtained by SCLC technique, as shown in Fig. 5. It may be because the effect of the trap was not considered before. In fact, there exhibits the discrete or shallow trap states in HAT-CN : NPB and HAT-CN : TAPC mixtures according to their $C-f$ characteristics. As we know, the trap states can greatly influence the charge carrier mobility and even the device performance. In addition, the hole mobilities slightly decrease with the electric field increasing, which is in agreement to our previous result. ${ }^{29}$ The phenomenon of the slightly decreased mobility is considered as to be originated from the presence of certain energetic disorder. ${ }^{32,33}$ When the descent of the electrostatic potential is comparable and even transcends the energy barrier, for an along-field jump, the dwell time of

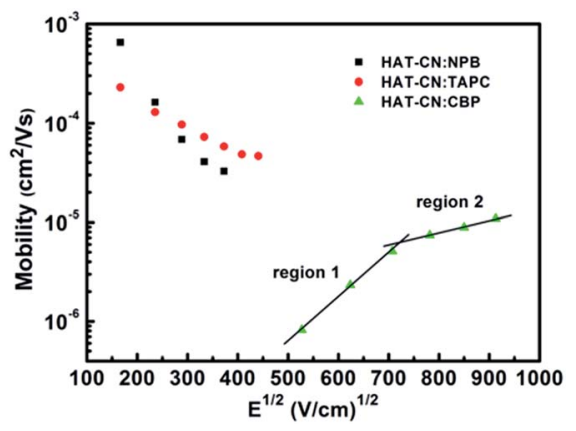

Fig. 4 Hole mobilities of HAT-CN : NPB, HAT-CN : TAPC and HAT$\mathrm{CN}$ : CBP mixtures against the square root of the electric-field strength.

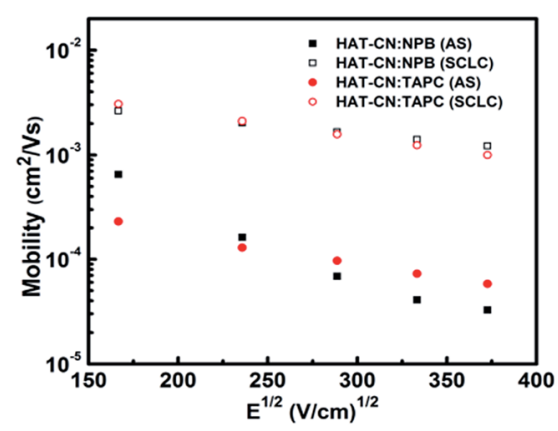

Fig. 5 Hole mobilities of HAT-CN : NPB and HAT-CN : TAPC mixtures against the square root of the electric-field strength obtained by AS and SCLC techniques, respectively. 
a carrier on a site approaches the reciprocal rate for that jump because the jump reverse the electric field direction are gradually eliminated. As a result, the transport velocity will saturate with the electric field. Correspondingly, the carrier $\tau_{\mathrm{dc}}$ does not change with the increment of applied voltage. This implies a linear decrease of the carrier mobility because it is controlled by energetically downward jump only that is not accelerated. For HAT-CN : CBP device, however, the hole mobility is in the range of $10^{-7}-10^{-5} \mathrm{~cm}^{2} \mathrm{~V}^{-1} \mathrm{~s}^{-1}$. The mobility curve demonstrates two linear parts. (1) In low electric field region, it can be seen that the mobility $\left(8.2 \times 10^{-7}-5.1 \times 10^{-6} \mathrm{~cm}^{2} \mathrm{~V}^{-1} \mathrm{~s}^{-1}\right)$ exhibits strong field-dependence and sharply increases as the applied electric field increases. (2) In high electric field region, the mobility $\left(7.4 \times 10^{-6}-1.1 \times 10^{-5} \mathrm{~cm}^{2} \mathrm{~V}^{-1} \mathrm{~s}^{-1}\right)$ slightly increases as the electric field increases and almost saturates. This increase should be the trapping effect of trap states. When the electric field increased, the trap depth can be decreased by Poole-Frenkel effect, leading to the increasing mobility. As shown in the inset in Fig. 1, at high applied bias voltage, the $J-V$ curve shows the conductance property of SCLC with exponential traps. This suggests that the hole transport in HAT-CN : CBP mixture is trap-controlled. It is clearly evident that the trapping effect leads to the much lower hole mobility.

\subsection{Holes transport mechanisms of mixtures}

3.3.1 Analysis of energy level. Fig. 6 shows the schematic energy diagram of the four pure semiconductor materials. Energy level values are taken from the literature. ${ }^{34-37}$ The highest occupied molecular orbital (HOMO) levels of NPB and TAPC are both $5.4 \mathrm{eV}$, which is only $0.3 \mathrm{eV}$ higher than the lowest unoccupied molecular orbital (LUMO) levels of HAT-CN (5.7 eV). Such energy level distribution can allow the electrons of NPB HOMO or TAPC HOMO to be easily excited to the HAT-CN LUMO, which is sometimes called charge carrier generation. As a consequence, the hole transport abilities of the two mixtures are strong. While the HOMO level of CBP is $6.0 \mathrm{eV}$, which is $0.3 \mathrm{eV}$ lower than the HAT-CN LUMO. Then the CBP molecule plays the role of traps in the hole transport process, which is also reflected in the $J-V$ characteristics of the HAT-CN : CBP device. The hole mobility of the device is very low due to the influence of traps.

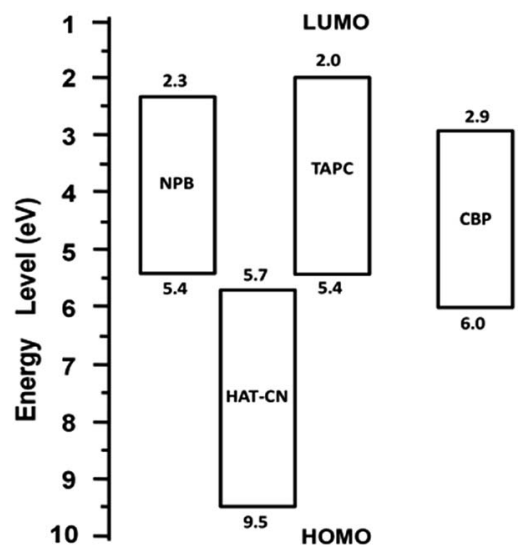

Fig. 6 Energy level diagram of HAT-CN, NPB, TAPC and CBP.

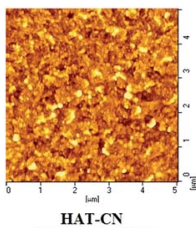

RMS (nm): 4.57

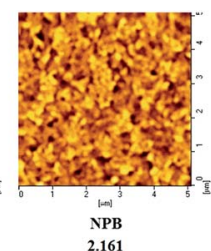

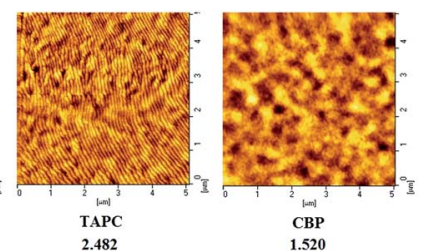

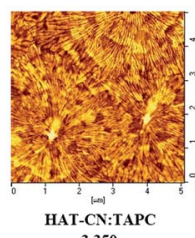

3.350

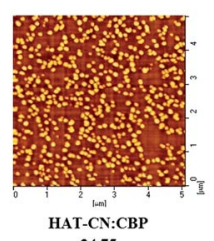

24.75
Fig. 7 The AFM images and roughness of HAT-CN, NPB, TAPC, CBP, HAT-CN : NPB, HAT-CN : TAPC and HAT-CN : CBP films.

3.3.2 Analysis of AFM. In order to further clarify the holes transport mechanisms in the mixed films, we studied the surface morphologies of the films before and after mixing by AFM. The results are shown in Fig. 7. The root mean square (RMS) of the pure films of HAT-CN, NPB, TAPC and CBP is $4.576 \mathrm{~nm}, 2.161 \mathrm{~nm}, 2.482 \mathrm{~nm}$ and $1.520 \mathrm{~nm}$, respectively. Clearly, in the four pure films, the RMS of CBP is the smallest. Thus, the RMS of the mixture film of HAT-CN : CBP is as high as $24.75 \mathrm{~nm}$, which is unfavorable for the hole transport. It is further explained the reason of the lowest holes mobility in HAT-CN : CBP device. On the contrary, the RMS of HAT$\mathrm{CN}$ : NPB and HAT-CN : TAPC is $3.025 \mathrm{~nm}$ and $3.350 \mathrm{~nm}$, respectively, which is relatively small and close. It is suggested that they have high and similar hole mobilities.

\section{Conclusions}

In summary, we have studied the hole transport characteristics and mechanisms of the three small molecule mixtures of HATCN : NPB, HAT-CN : TAPC and HAT-CN : CBP. The currentvoltage characteristics show that the holes conductions are both SCLC. The hole mobilites of HAT-CN : NPB and HAT-CN : TAPC are determined to be orders of $\sim 10^{-4} \mathrm{~cm}^{2} \mathrm{~V}^{-1} \mathrm{~s}^{-1}$ by AS measurements, which slightly decrease as the electric field increases. Nevertheless, the hole transport in HAT-CN : CBP is SCLC with exponential distribution traps. The hole mobility of HAT-CN : CBP is obtained from $8.2 \times 10^{-7}$ to $1.1 \times 10^{-5} \mathrm{~cm}^{2}$ $\mathrm{V}^{-1} \mathrm{~s}^{-1}$, which shows two regions. At low electric field region, the hole mobility $\left(8.2 \times 10^{-7}-5.1 \times 10^{-6} \mathrm{~cm}^{2} \mathrm{~V}^{-1} \mathrm{~s}^{-1}\right)$ sharply increases with increasing the electric field. Whereas at high electric field region, the mobility $\left(7.4 \times 10^{-6}-1.1 \times 10^{-5} \mathrm{~cm}^{2}\right.$ $\mathrm{V}^{-1} \mathrm{~s}^{-1}$ ) slightly increases with increasing the electric field, and almost reaches saturate with the electric field, which should result from the trapping of trap states. Furthermore, the energy level analysis suggests that there are charge carrier generation in HAT-CN : NPB and HAT-CN : TAPC. The CBP molecule acts as a trap during the hole transport process. AFM images clearly show the film surfaces of HAT-CN : NPB and HAT-CN : TAPC mixtures are relatively smooth, which is beneficial to the hole transport. The HAT-CN : CBP film exhibits much rough, 
indicating the poor hole transport ability. Our results further demonstrate that AS is a simple and powerful tool for the characterization of charge carrier transport properties in organic semiconductor mixtures. We believe that our results will provide critical theoretical information for material selection, device structure design and performance analysis.

\section{Acknowledgements}

The authors gratefully acknowledge the National Natural Science Foundation of China (51333007, 91433201, 61204059), Ministry of Science and Technology of China (973 program No. 2013CB834805), the Foundation of Jilin Research Council (2012ZDGG001, 20130206003GX), Chinese Academy of Sciences (KGZD-EW-303-3), Scientific Research Foundation of the Education Department of Shaanxi Province, China (15JK1849) for the support of this research.

\section{References}

1 G. Yu, J. Gao, J. C. Hummelen and A. J. Heeger, Science, 1995, 270, 1789-1791.

2 L. S. Liao and K. P. Klubek, Appl. Phys. Lett., 2008, 92, 223311.

3 W. S. Jeon, J. S. Park, L. Li, D. C. Lim, Y. H. Son, M. C. Suh and J. H. Kwon, Org. Electron., 2012, 13, 939-944.

4 Q. X. Guo, H. D. Sun, J. X. Wang, D. Z. Yang, J. S. Chen and D. G. Ma, J. Mater. Chem. C, 2016, 4, 376-382.

5 H. D. Sun, Q. X. Guo, D. Z. Yang, Y. H. Chen, J. S. Chen and D. G. Ma, ACS Photonics, 2015, 2, 271-279.

6 L. Burtone, D. Ray, K. Leo and M. Riede, J. Appl. Phys., 2012, 111, 064503.

7 N. Gasparini, S. Righi, F. Tinti, A. Savoini, A. Cominetti, R. Po and N. Camaioni, ACS Appl. Mater. Interfaces, 2014, 6, 2141621425.

8 W. L. Ma, C. Y. Yang, X. Gong, K. Lee and A. J. Heeger, Adv. Funct. Mater., 2005, 15, 1617-1622.

9 M. Redecker, D. D. C. Bradley, M. Inbasekaran and E. P. Woo, Appl. Phys. Lett., 1998, 73, 1565.

10 A. J. Campbell, D. D. C. Bradley and H. Antoniadis, J. Appl. Phys., 2001, 89, 3343.

11 P. W. M. Blom and M. C. J. M. Vissenberg, Phys. Rev. Lett., 1998, 80, 3819.

12 S. C. Tse, H. H. Fong and S. K. So, J. Appl. Phys., 2003, 94, 2033.

13 D. Poplavskyy, J. Nelson and D. D. C. Bradley, Appl. Phys. Lett., 2003, 83, 707.

14 N. L. Hong, X. Q. Qiu, W. Y. Deng, Z. C. He and Y. Li, RSC Adv., 2015, 5, 90913-90921.

15 P. Anjaneyulu, C. S. Suchand Sangeeth and R. Menon, J. Appl. Phys., 2010, 107, 093716.
16 M. Hoping, C. Schildknecht, H. Gargouri, T. Riedl, M. Tilgner, H. H. Johannes and W. Kowalsky, Appl. Phys. Lett., 2008, 92, 213306.

17 S. Bellani, A. Iacchetti, M. Porro, L. Beverina, M. R. Antognazza and D. Natali, Org. Electron., 2015, 22, 56-61.

18 S. Ishihara, H. Hase, T. Okachi and H. Naito, Org. Electron., 2011, 12, 1364-1369.

19 S. W. Tsang, S. C. Tse, K. L. Tong and S. K. So, Org. Electron., 2006, 7, 474-479.

20 E. Ehrenfreund, C. Lungenschmied, G. Dennler, H. Neugebauer and N. S. Sariciftci, Appl. Phys. Lett., 2007, 91, 012112.

21 K. H. Chan and S. K. So, J. Photonics Energy, 2011, 1, 011011. 22 J. You, L. Dou, K. Yoshimura, T. Kato, K. Ohya, T. Moriarty, K. Emery, C. C. Chen, J. Gao, G. Li and Y. Yang, Nat. Commun., 2013, 4, 1446.

23 Y.-K. Kim, K. J. Won and Y. Park, Appl. Phys. Lett., 2009, 94, 063305.

24 P. S. Szalay, J. R. Galan-Mascaros, B. L. Schottel, J. Bacsa, L. M. Perez, A. S. Ichimura, A. Chouai and K. R. Dunbar, J. Cluster Sci., 2004, 15, 503-530.

25 P. Frank, N. Koch, M. Koini, R. Rieger, K. Müllen, R. Resel and A. Winkler, Chem. Phys. Lett., 2009, 473, 321.

26 H. Glowatzki, B. Broker, R. P. Blum, O. T. Hofmann, A. Vollmer, R. Rieger, K. Mullen, E. Zojer, J. P. Rabe and N. Koch, Nano Lett., 2008, 8, 3825-3829.

27 L. S. Liao and K. P. Klubek, Appl. Phys. Lett., 2008, 92, 223311. 28 D. H. Kim and T. W. Kim, Org. Electron., 2014, 15, 3452-3457. 29 Y. P. Wang, J. Y. Huang, J. S. Chen, X. F. Qiao, D. Z. Yang, D. G. Ma and L. S. Dong, Chin. Phys. Lett., 2016, 33, 027302. 30 S. W. Tsang, S. K. So and J. B. Xu, J. Appl. Phys., 2006, 99, 013706.

31 J. Shao and G. T. Wright, Solid-State Electron., 1961, 3, 291303.

32 A. J. Mozer, N. S. Sariciftci, A. Pivrikas, R. Österbacka, G. Juška, L. Brassat and H. Bässler, Phys. Rev. B: Condens. Matter Mater. Phys., 2005, 71, 035214.

33 K. Seki and M. Tachiya, Phys. Rev. B: Condens. Matter Mater. Phys., 2001, 65, 014305.

34 K. S. Yook, S. O. Jeon and J. Y. Lee, Thin Solid Films, 2009, 517, 6109-6111.

35 K. Goushi, R. Kwong, J. J. Brown, H. Sasabe and C. Adachi, J. Appl. Phys., 2004, 95, 7798.

36 J. Wünsche, S. Reineke, B. Lüssem and K. Leo, Phys. Rev. B: Condens. Matter Mater. Phys., 2010, 81, 245201.

37 M. E. Kondakova, T. D. Pawlik, R. H. Young, D. J. Giesen, D. Y. Kondakov, C. T. Brown, J. C. Deaton, J. R. Lenhard and K. P. Klubek, J. Appl. Phys., 2008, 104, 094501. 